Revista de Investigación Educativa 26

enero-junio, 2018 | ISSN 1870-5308 | Xalapa, Veracruz

Instituto de Investigaciones en Educación | Universidad Veracruzana

\title{
¿Qué estado guarda la educación indígena en Veracruz?
}

Jessica Badillo Guzmána

Recibido: 27 de noviembre de 2017

Aceptado: 08 de diciembre de 2017

Niembro, M. C. \& Mendoza, R. G. (2017). La educación indígena en Veracruz: diagnóstico y recomendaciones para la política educativa. Xalapa: Universidad Veracruzana. 127 págs. ISBN: 978-607-502-581-0.

$\mathrm{H}$

istóricamente, los indígenas en México han sido uno de los sectores menos favorecidos por la política pública, lo que ha generado una gran asimetría entre las condiciones de vida de ese sector frente al resto de la población. Sea que se ubiquen en el medio rural o en el urbano, su acceso a servicios de salud, vivienda y educación es limitado, además de que su atención en los sistemas de justicia es deficiente y su patrimonio biocultural es cada vez más afectado. Todo ello genera contextos marcados además por la desigualdad, el racismo y la discriminación, que repercuten negativamente en el desarrollo socioeconómico de los pueblos originarios.

La educación es una palanca para el desarrollo de las sociedades, y en el caso de la población indígena lo es aún más, no únicamente por la posibilidad que brinda de acceder a oportunidades de movilidad social, sino porque representa una puerta 
abierta para el conocimiento de sus derechos y para su defensa, para posicionarse en el mundo desde su propia escritura y lectura del mismo, desde su cosmovisión. Por ello, generar conocimiento sobre la realidad indígena de nuestro país en materia educativa es un ejercicio necesario, y por ello también el libro que aquí se reseña es una lectura obligada para quienes se interesan en el análisis de este campo a través de trabajos de investigación, pero también para quienes se encargan de la toma de decisiones en la política educativa estatal.

En La educación indígena en Veracruz: diagnóstico y recomendaciones para la política educativa, ${ }^{1}$ María Consuelo Niembro Domínguez y Rosa Guadalupe Mendoza Zuany nos ofrecen una caracterización amplia del estado que guarda la educación indígena en Veracruz, además de proporcionar, a partir del análisis efectuado, un conjunto de recomendaciones para la política pública regional, que en más de un caso, bien podrían adecuarse para la mejora de la política de alcance nacional, pues la población indígena en nuestro país comparte problemáticas, aunque en escalas diversas $y$ en contextos diferenciados.

Niembro Domínguez ha sido asesora técnico pedagógica en la Dirección de Educación Indígena de la Secretaría de Educación de Veracruz, y hasta enero de 2017 fue Jefa del Departamento de Operación de Programas Técnico Pedagógicos de Educación Indígena de dicha dependencia. Su amplia experiencia en este ámbito, su mirada crítica y propositiva y su perfil como antropóloga social constituyen sin duda referentes fundamentales para realizar un examen de la educación indígena "desde dentro". Mendoza Zuany, por su parte, investigadora del Instituto de Investigaciones en Educación de la Universidad Veracruzana y doctorada en Políticas por la Universidad de York, es especialista en evaluación de políticas educativas en educación intercultural e indígena, por lo que su visión sobre la educación indígena "desde fuera" contribuye a la generación de un balance necesario y pertinente sobre este ámbito. Desde estas miradas que se complementan, Niembro y Mendoza acercan a los lectores al estado que guarda la educación indígena en Veracruz.

La introducción del libro, además de presentarlo de manera general y señalar las fuentes consultadas para la investigación: "documentos públicos oficiales, estadísticas, evaluaciones de políticas educativas, grupos de discusión con docentes del subsistema indígena en Veracruz y académicos que se dedican a la investigación educativa” (p. 7), recupera buena parte del marco legal para la atención educativa de la

1. El libro cuenta con una versión electrónica que puede consultarse en https://www.uv.mx/bdie/ 
población indígena en México, así como referentes de ésta en la entidad veracruzana. Aborda también los resultados de la Consulta Previa, Libre e Informada a Pueblos y Comunidades Indígenas sobre Evaluación Educativa, realizada en 2015 por el Instituto Nacional para la Evaluación de la Educación (INEE). Para finalizar, las autoras describen el enfoque inclusivo con perspectiva intercultural bilingüe que debe sustentar las prácticas en educación indígena.

A la introducción le siguen ocho capítulos que corresponden a los ámbitos de análisis que las autoras trabajaron: características generales del subsistema, perfil docente, formación continua, pertinencia cultural del curriculum, lengua indígena, materiales educativos, infraestructura y equipamiento y gestión escolar. En cada uno de ellos el lector encontrará, en un primer momento, un diagnóstico apoyado en estadísticas estatales - en algunos casos en comparación con las nacionales-, así como una reflexión crítica sobre las transformaciones que se han impulsado a partir de programas estratégicos derivados de políticas regionales o nacionales. En un segundo momento, las autoras presentan un conjunto de recomendaciones con base en el análisis realizado.

En general, los diagnósticos brindan una visión sucinta de la realidad que vive la entidad veracruzana en educación indígena; nos muestran, por un lado, las desigualdades que este subsistema presenta, el reto de la profesionalización y la educación continua de los docentes, el rezago en los resultados de aprendizaje y los esfuerzos para abatirlo, la problemática de la pertinencia cultural y lingüística del curriculum, de los materiales educativos y del perfil de los profesores, la necesidad de fortalecer la gestión escolar y mejorar las condiciones de infraestructura y equipamiento de las escuelas indígenas — quizás las más olvidadas en el sistema educativo nacional-; por otro lado, destacan los avances que se han logrado en cada ámbito, así como las dificultades que la misma Dirección de Educación Indígena enfrenta para avanzar en su misión y las acciones que ha tomado para hacerles frente.

En una época en la que la educación en México es cuestionada debido a las reformas recientemente implementadas, al escaso presupuesto dedicado a este sector $y$ a los deficientes resultados de aprendizaje, en la que se coloca la responsabilidad de los resultados educativos en los docentes y se pasan por alto todas las problemáticas socioeconómicas que aquejan a la población y que repercuten en la vida escolar, $L a$ educación indígena en Veracruz: diagnóstico y recomendaciones para la política educativa nos recuerda que mientras se reconozca pero no se atienda con pertinencia la diversidad cultural y lingüística, mientras las políticas educativas no sean dirigidas 
a los espacios con mayores necesidades y carencias, mientras no exista coordinación entre las dependencias estatales y nacionales, y mientras no haya voluntad política para sacar del rezago a las comunidades originarias del país, los esfuerzos en materia de educación indígena seguirán siendo aislados, y la población étnica, discriminada sistémicamente. En ello radica también su importancia. 